\title{
LETTER \\ UMMS: Efficient Superpixel Segmentation Driven by a Mixture of Spatially Constrained Uniform Distribution
}

\author{
Pengyu WANG ${ }^{\dagger}$, Hongqing $\mathrm{ZHU}^{\dagger}{ }^{\text {a) }}$, and Ning CHEN ${ }^{\dagger}$, Members
}

\begin{abstract}
SUMMARY A novel superpixel segmentation approach driven by uniform mixture model with spatially constrained (UMMS) is proposed. Under this algorithm, each observation, i.e. pixel is first represented as a fivedimensional vector which consists of colour in CLELAB space and position information. And then, we define a new uniform distribution through adding pixel position, so that this distribution can describe each pixel in input image. Applied weighted 1-Norm to difference between pixels and mean to control the compactness of superpixel. In addition, an effective parameter estimation scheme is introduced to reduce computational complexity. Specifically, the invariant prior probability and parameter range restrict the locality of superpixels, and the robust mean optimization technique ensures the accuracy of superpixel boundaries. Finally, each defined uniform distribution is associated with a superpixel and the proposed UMMS successfully implements superpixel segmentation. The experiments on BSDS500 dataset verify that UMMS outperforms most of the state-of-theart approaches in terms of segmentation accuracy, regularity, and rapidity. key words: uniform mixture model, spatially constrained, weighted 1Norm, superpixel segmentation
\end{abstract}

\section{Introduction}

The essence of superpixel segmentation is to partition an image into a number of connected and unified pixel groups with perceptual significance. Therefore, superpixels should be more consistent with human visual cognition. It not only extracts visual features conveniently, but also greatly reduces computational complexity of the algorithm [1], [2]. Generally, superpixel segmentation algorithm should meet boundary adherence, pixel similarity, and superpixel regularity, etc. Regular superpixels can maintain the typical characteristics of the spatial topology, structure, homogeneity and isometric information of the included pixels, and help subsequent algorithms to extract local features. Recently, Liu et al. [3] introduced a clustering objective function (ERS) based on entropy rate of a random walk and a equilibrium term. It helps to generate superpixels with high boundary recall and segmentation precision, but these superpixels have irregular shape and rough border. SLIC [4] applied $k$-means clustering method to generate superpixels. SLIC was verified to produce better adherence to image boundary. SLIC runs for a significantly short time. Machairas et al. [5] introduced the waterpixels strategy for

\section{Manuscript received July 9, 2019.}

Manuscript revised September 5, 2019.

Manuscript publicized October 2, 2019.

$\dagger$ The authors are with the School of Information Science and Engineering, East China University of Science and Technology, Shanghai 200237, China.

a) E-mail: hqzhu@ecust.edu.cn

DOI: 10.1587/transinf.2019EDL8134 superpixel segmentation which bases on watershed transformation. LRW [6] could generate fairly regular superpixels with relatively high precision, but the computation is complicated. For LSC [7], it has advantage in running time, accuracy and regularity. Compared with the above approaches, GMMS [8] has been proved to have the highest boundary recall, accuracy, and relatively high running speed, but the shape of generated superpixels is very irregular.

In this paper, we propose a new superpixel segmentation model derived by uniform mixture model with spatially constrained (UMMS). To the best of the authors' knowledge, to date, no uniform mixture model has been reported in the literature for deriving superpixel generation. The characteristics of our approach could be summarized as: (i) defining a novel uniform distribution; (ii) considering intensity and coordinate of each pixel, so that the defined uniform distribution can describe all pixels of image; (iii) providing a fast and robust parameter estimation scheme, where the parameter range and priori probability of UMMS are fixed. This parameter estimation method greatly reduces computing costs; (iv) weighted 1-Norm balances the colour similarity and spatial proximity. In this way, the compactness of superpixels can be well controlled. These characteristics make UMMS do well in superpixel segmentation. The accuracy and rapidity of UMMS are later verified by experiments.

\section{Proposed Scheme}

The implementation of UMMS includes: defining a new uniform distribution, parameter initialization, parameter estimation, superpixel segmentation, and superpixel refinement. Figure 1 exhibits a block diagram of the proposed

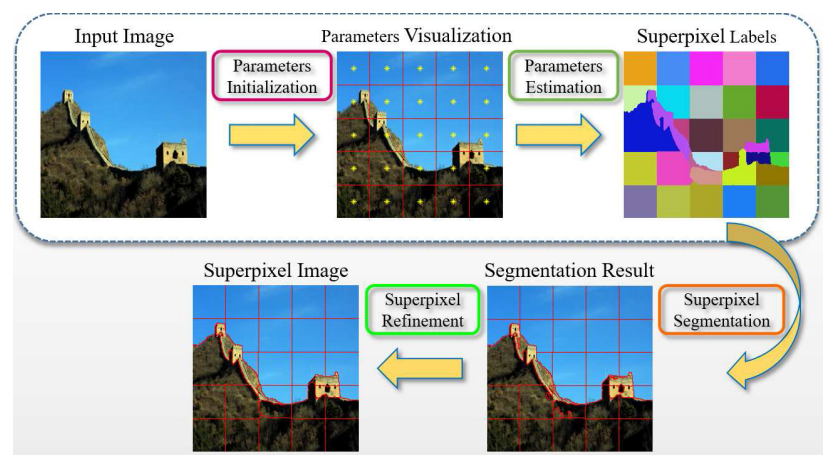

Fig. 1 Block diagram of the proposed superpixel segmentation scheme. 
scheme.

\subsection{Defining Uniform Distribution}

Our method begins via defining uniform distribution, and then constructs a uniform mixture model with spatial constraints to derive superpixel segmentation. Let $i$ represent the pixel index of an image $I$ of $h$ in height and $w$ in width. The proposed approach performs on the CLELAB colour space $\left(l_{i}, a_{i}, b_{i}\right)$. Here, let five-dimensional vector $z_{i}=\left(l_{i}, a_{i}, b_{i}, x_{i}, y_{i}\right)^{T}$ describe pixel $i$, where $\left(x_{i}, y_{i}\right)$ denotes the position of pixel $i, i=(1,2, \ldots, N)$, and $N$ is the number of pixels. Supposed that $n_{x}$ and $n_{y}$ are nodes with horizontal and vertical directions, the number of superpixel $K$ can be expressed as

$$
n_{x}=w / v_{x}, \quad n_{y}=h / v_{y}, K=n_{x} \cdot n_{y}
$$

where $w \bmod v_{x}=0$ and $h \bmod v_{y}=0$. Set $v_{x}=v_{y}$ to produce roughly equally sized superpixels.

The probability density function for a uniform distribution [9] on interval $[a, b]$ is defined as

$$
P(x)=\left\{\begin{array}{cl}
\frac{1}{b-a}, & a \leq x \leq b \\
0, & \text { others }
\end{array}\right.
$$

The advantage of classical uniform distribution is simplicity and intuition. However, the mixture model with this distribution could not describe different shapes of observed data. One example with the histogram of a natural image is depicted in Fig. 2. It can be visualized that the classical uniform distribution will not always exactly fit all pixels. More specifically, pixels with low probability distribution aren't grouped by the component of uniform mixture (see the place indicated by red arrow).

To overcome the above drawbacks, this study defines a new uniform distribution on basis of (2) as follows.

$$
U\left(z_{i} \mid \theta_{j}\right)=\left\{\begin{array}{l}
1, \quad \mu_{j}-\frac{r_{j}}{2} \leq z_{i} \leq \mu_{j}+\frac{r_{j}}{2} \\
\exp \left[(\alpha-1)\left\|z_{i, s}-\mu_{j, s}\right\|_{1}\right. \\
\left.-\alpha\left\|z_{i, c}-\mu_{j, c}\right\|_{1}\right], \quad \text { others }
\end{array}\right.
$$

where mean $\mu_{j}=\left(l_{j}, a_{j}, b_{j}, x_{j}, y_{j}\right), j=(1,2, \ldots, K)$, is defined as a five-dimensional vector containing colour and spatial information to match the observation $z_{i}$. Parameter $\theta_{j}=\left\{\mu_{j}, r_{j}\right\}, \mu_{j}$ and $r_{j}$ represent mean and range, respectively. $z_{i, c}=\left(l_{i}, a_{i}, b_{i}\right)$ and $z_{i, s}=\left(x_{i}, y_{i}\right)$ denote the colour and spatial components of the $i$-th pixel, respectively. Similarly, $\mu_{j, c}=\left(l_{j}, a_{j}, b_{j}\right)$ and $\mu_{j, s}=\left(x_{j}, y_{j}\right)$ are the colour and spatial components of mean with respect to the $j$-th label. $\|\cdot\|_{1}$ represents 1 -Norm.

In proposed uniform distribution, if $\mu_{j}-r_{j} / 2 \leq z_{i} \leq$ $\mu_{j}+r_{j} / 2$, the proposed uniform distribution value is 1 . From definition (3), for other cases, $U\left(z_{i} \mid \theta_{j}\right)$ is an exponential function of weighted 1-Norm between the observation $z_{i}$ and the mean $\mu_{j}$. Parameter $\alpha(\alpha \in[0,1])$ balances the tradeoff between the color similarity and spatial proximity. The bigger the parameter $\alpha$ is, the more significant colour information impacts in superpixel segmentation. While spatial information is more dominated when parameter $\alpha$ becoming small. That is, we will see more regular superpixel when $\alpha$ tends to be small. Such a definition guarantees that the distribution obtained has the ability to model all observed data with different regions. Details about these analyses are well validated in Fig. 2. For instance, observation $x=150$ could be described by proposed distribution Fig. 2 (see the place indicated by red star).

To achieve superpixel segmentation, UMMS assumes that each observation $z_{i}$ has an independent superpixels label $\Omega_{j}$, then, the probability density function is given by

$$
p\left(z_{i} \mid \Pi, \Theta\right)=\sum_{j=1}^{K} \pi_{j} U\left(z_{i} \mid \theta_{j}\right)
$$

where $\Theta=\left\{\theta_{j}\right\}$ means the model parameter, and $\Pi=\left\{\pi_{j}\right\}$ is the prior probability that should satisfy the constraints.

$$
0 \leq \pi_{j} \leq 1 \quad \text { and } \quad \sum_{j=1}^{K} \pi_{j}=1
$$

\subsection{Parameter Initialization}

Since the aim of our method is to generate superpixels with uniform size as much as possible, which means each superpixel has to own approximately the same number of pixels. Therefore, the fixed prior probability $\pi_{j}$ is used for each uniform distribution

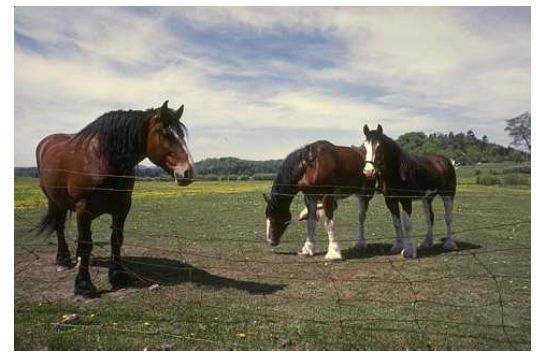

(a)

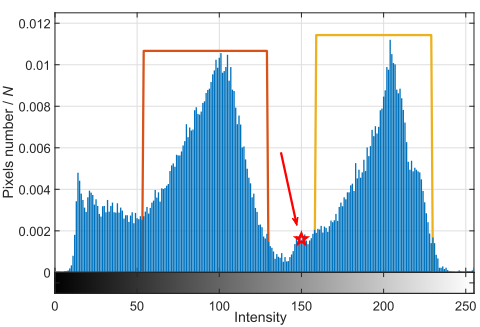

(b)

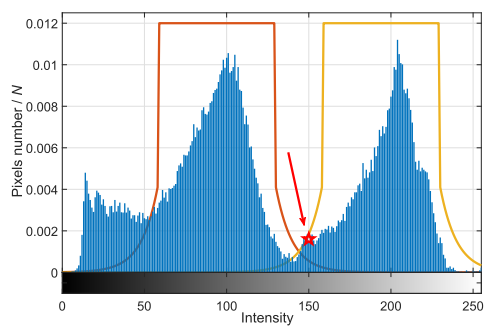

(c)

Fig. 2 Comparison of the distributions, (a) original image; (b) using classical uniform distribution; (c) using our defined uniform distribution 


$$
\pi_{j}=1 / K, j=(1,2, \ldots, K)
$$

For the mean of the $j$-th superpixel, its colour component indicates the average colour of the certain pixel neighborhoods, and its spatial component correspond to the coordinate information of the certain pixel as well. The index of this pixel is calculated by

$$
d=j_{x} \cdot v_{x}+\left(v_{x} / 2\right)+w \cdot\left[j_{y} \cdot v_{y}+\left(v_{x} / 2\right)\right]
$$

where $j_{x}=j \bmod n_{x}$ and $j_{y}=j / n_{x}$. For the colour component $\mu_{j, c}$ of mean $\mu_{j}$ can be computed as

$$
\mu_{j, c}\left(l_{j}, a_{j}, b_{j}\right)=\frac{1}{T} \sum_{t \in \partial_{d}} z_{t, c}\left(l_{t}, a_{t}, b_{t}\right)
$$

where $T$ stands for the number of neighboring pixels around the observation $z_{d}$. And a square window of size $T=7 \times 7$ is used in this paper. $\partial_{d}$ is the neighborhood of the $d$-th observation. The spatial component $\mu_{j, s}$ of mean $\mu_{j}$ is given by

$$
\mu_{j, s}\left(x_{j}, y_{j}\right)=z_{d, s}\left(x_{d}, y_{d}\right)
$$

Parameter range $r_{j}$ is set to $v_{x}$ to reduce computational cost.

\subsection{Parameter Estimation}

According to the above description, we summarize the parameter estimation in Algorithm 1,

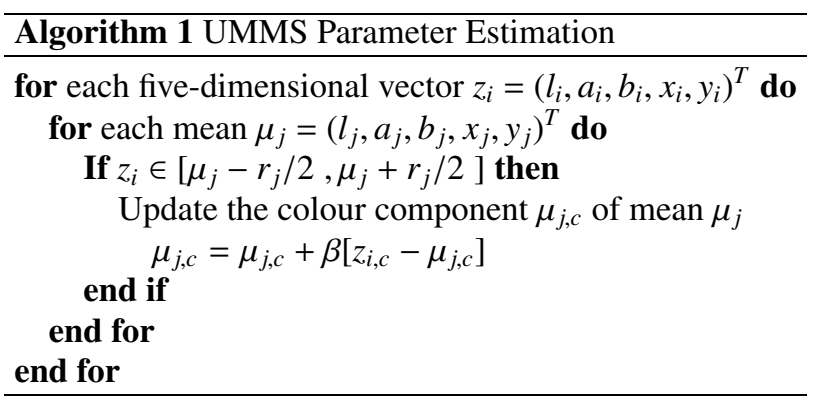

where $\beta \in[0,1]$ is the learning rate. Finally, we have the following posterior probability $R_{i j}$

$$
R_{i j}=\frac{U\left(z_{i} \mid \mu_{j}, r_{j}\right)}{\sum_{k \in K} U\left(z_{i} \mid \mu_{k}, r_{k}\right)}
$$

Once the parameter estimation phase is completed, each pixel is assigned a superpixel label with the maximum posterior probability $R_{i j}$.

\subsection{Superpixel Segmentation and Refinement}

To have our approach explicitly force the connection along boundaries, some considerations should factor into the quality of our superpixel segmentation model. Here, we add a postprocessing step used by SLIC algorithm [4]. This step is summarized below: (i) superpixel, which size is smaller than $v_{x}$ pixels, should be merged into other adjacent superpixels in terms of colour information; (ii) impose a morphological closing operation on each superpixel, and subtract the original superpixel from its result. The obtained pixels are reallocated to the nearest superpixels to smooth boundary.

\section{Experimental Results}

The experiment compares UMMS with five advanced superpixel segmentation methods named $\operatorname{ERS}^{\dagger}[3], \operatorname{SLIC}^{\dagger \dagger}[4]$, $\mathrm{LRW}^{\dagger \dagger \dagger}$ [6], $\mathrm{LSC}^{\dagger+\dagger}$ [7], and GMMS ${ }^{\dagger+\dagger \dagger}$ [8] on BSDS500 dataset [10]. For all the five approaches, the implementations are based on publicly available online source code.

Parameter $\alpha$ is assigned to 0.8 , and $\beta=1 / N$. All simulations were carried out on a desktop computer with an Intel $4.1 \mathrm{GHz}$ hexa core processor and 16GB of RAM. Each algorithm is developed in Windows 10 using MATLAB 2018a. The source code and test images used in our model are available at website ${ }^{\dagger+1+\dagger \dagger}$. The evaluation of experimental result is conducted via boundary recall (BR) [7], under-segmentation error (UE) [7], achievable segmentation accuracy (ASA) [8], and weighted isoperimetric quotient (WIPQ) [8].

The segmentation results could be seen in Fig. 3. An area of interest is amplified to illustrate further details as shown in Fig. 4. Each method extracts about 180 superpixels. Figure 5 shows the experimental results of different superpixels, where each image is segmented approximately into $1500 \backslash 700$ superpixels. As indicated by these results of visual comparison, ERS shows delicate segmentation details, but because of the rough boundary, the overall visual effect is the worst. The superpixels of LRW and SLIC have relatively regular shapes, whereas the poor local detail makes them less visually pleasing. Compared with the above three algorithms, LSC and GMMS exhibit considerably competitive visual effect, although sometimes they still have some over-segmentation or under-segmentation. UMMS has achieved generally better results in terms of visual effect.

To get a comprehensive and objective evaluation, the experiment compares the proposed method with five state-of-the-art algorithms on BSDS500 test dataset, which consists of 200 test images with the manually labeled groundtruth. Here, we provided an additional experiment to assess the effect of the weighting on the segmentation performance. For the purpose of illustration, the segmentation accuracy of UMMS without weight is compared with that of other algorithms. We compute the average BR, UE, ASA, and WIPQ of each model. Figure 6 displays the quan-

\footnotetext{
${ }^{\dagger}$ https://github.com/mingyuliutw/ers

${ }^{\dagger} h \mathrm{http}$ ///ivrl.epfl.ch/research/superpixels

th https://github.com/shenjianbing/lrw14

t† http://jschenthu.weebly.com/projects.html

t+t https://github.com/ahban/GMMSP

${ }^{\dagger+\dagger+\dagger} h$ ttp://www.imagetech-polynomials.com/Superpixel.html
} 

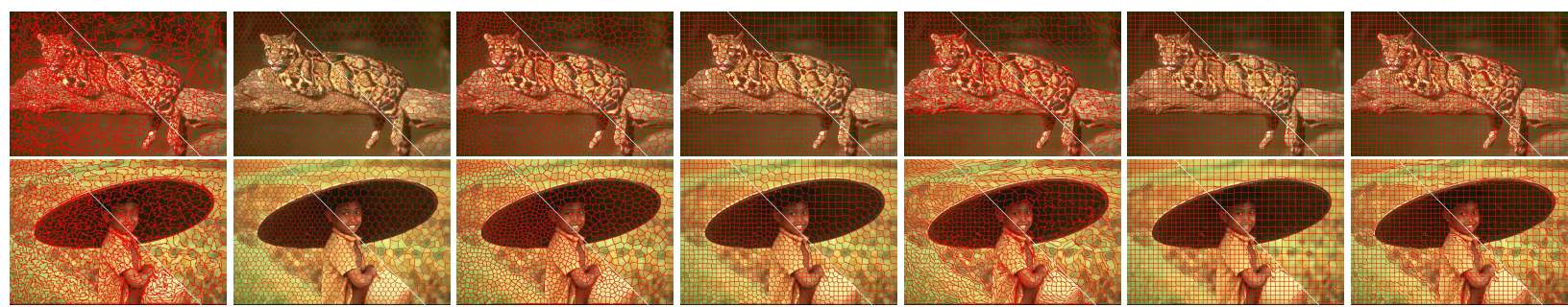

Fig. 5 The images are segmented approximately into $1500 \backslash 700$ superpixels, from the first column to the last is ERS, SLIC, LRW, LSC, GMMS, UMMS (unweighted), and UMMS, respectively
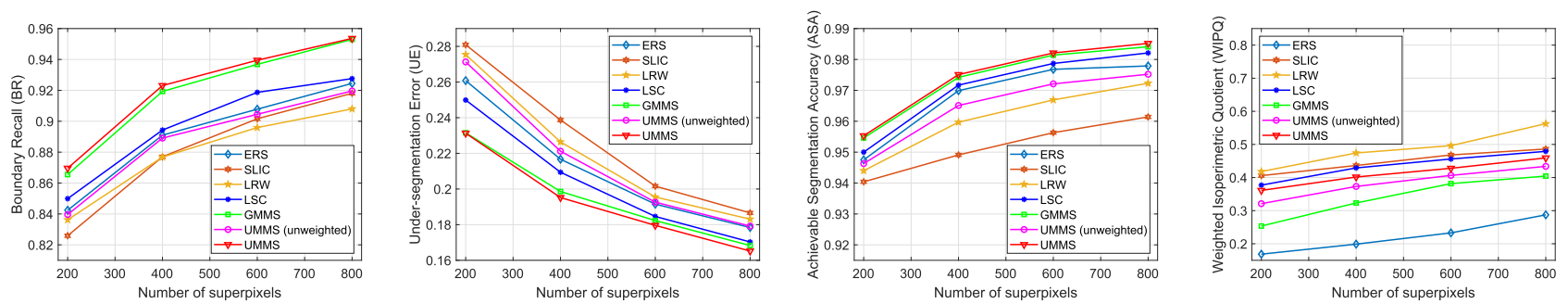

Fig. 6 Comparison with other algorithms in BSDS500 test dataset.
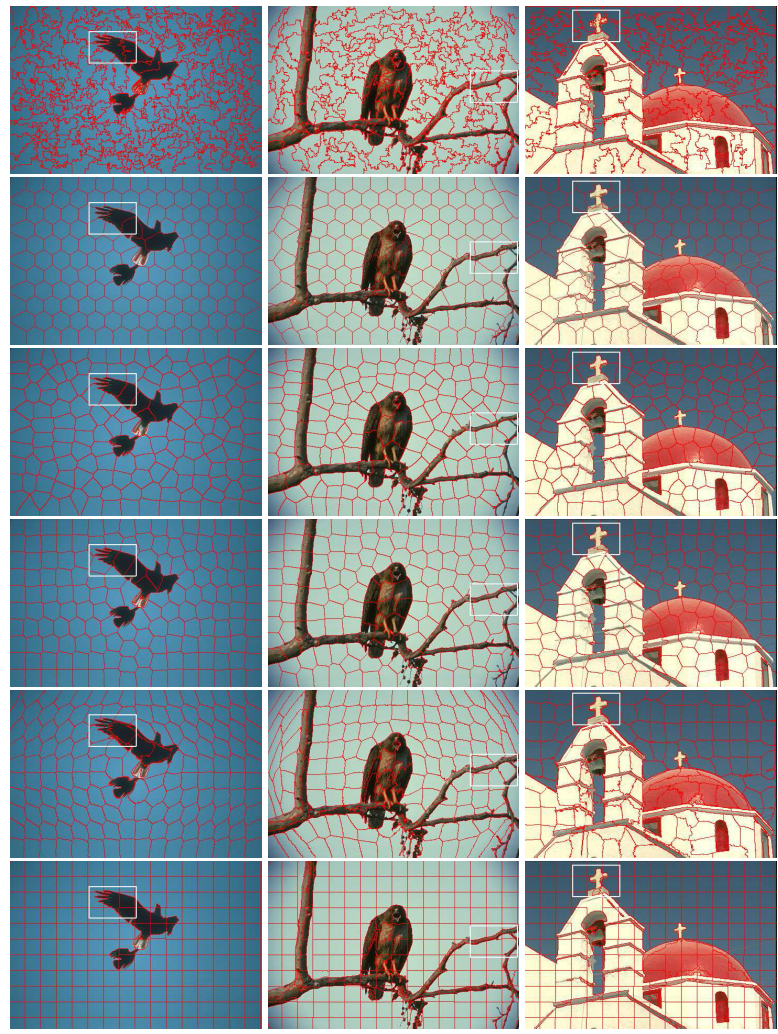

Fig. 3 Superpixel segmentation, from the first row to the last is ERS, SLIC, LRW, LSC, GMMS, and UMMS, respectively

titative evaluation of all approaches use these four metrics. The superpixel numbers are set to 200, 400,600, and 800, respectively. The segmentation accuracy comparison results show: (i) according to regularity evaluation, our method outperforms GMMS and ERS in terms of regularity evaluation metric WIPQ; (ii) according to accuracy evaluation, the pro-

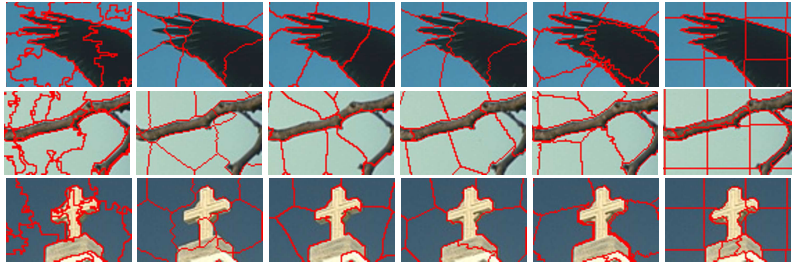

Fig. 4 Visual comparison of detailed parts, from the first column to the last is ERS, SLIC, LRW, LSC, GMMS, and UMMS, respectively.

posed UMMS is superior to the selected methods in terms of BR, UE, and ASA; (iii) we found that weighted UMMS performed better than unweighted UMMS, so we concluded that weighting factor was necessary for the new defined uniform distribution.

The last experiment investigates computational complexity of UMMS. The main time-consuming part of UMMS includes the updating of mean and posterior probability, (i) because the prior probability $\pi_{j}$ and the range $r_{j}$ are fixed, only the mean $\mu_{j}$ needs to be updated. Therefore, if each initial superpixel size is $v_{x} \times v_{y}$, with a total of $n_{x} \times n_{y}$ superpixels. Given $\left(v_{x} \times v_{y}\right) \cdot\left(n_{x} \times n_{y}\right)=h \times w=N$, so the computational complexity is $O(N)$, (ii) because most of the pixels belong to the threshold interval with uniform coordinate distribution, the posterior probability of these pixels can be directly assigned a value. The computational complexity for this operation is $O(N)$. Only few pixels need to calculate the weighted 1-Norm. The time complexity for computing it is $O(K \times N)$. Clearly, updating posterior probability has linear complexity. In addition, the computational cost of superpixels refinement is relatively small. Therefore, the total computational complexity of UMMS is $O(N)$.

Finally, we investigate the time required for each superpixel approach to segment images. Table 1 reports their average execution time on the BSDS500 test dataset. As 
Table 1 Comparison with other algorithms in run-times (seconds).

\begin{tabular}{ccccccc}
\hline Superpixels & ERS & SLIC & LRW & LSC & GMMS & UMMS \\
\hline 100 & 2.683 & 0.512 & 53.752 & 0.913 & 0.825 & 0.494 \\
200 & 2.701 & 0.525 & 99.604 & 0.924 & 0.827 & 0.508 \\
300 & 2.717 & 0.541 & 122.364 & 0.936 & 0.828 & 0.513 \\
400 & 2.732 & 0.553 & 155.963 & 0.947 & 0.830 & 0.524 \\
500 & 2.741 & 0.568 & 201.585 & 0.955 & 0.832 & 0.536 \\
600 & 2.756 & 0.576 & 247.691 & 0.961 & 0.836 & 0.550 \\
700 & 2.785 & 0.587 & 296.388 & 0.969 & 0.839 & 0.573 \\
800 & 2.799 & 0.609 & 335.295 & 0.978 & 0.842 & 0.589 \\
\hline
\end{tabular}

shown in the table, SLIC and UMMS perform much faster than LRW. Our algorithm outperforms most algorithms in execution time. A more detailed comparison of the computation time is shown in Table 1.

\section{Conclusion}

This paper developed an efficient superpixel segmentation approach driven by uniform mixture model with spatially constrained. In this scheme, we defined a novel uniform distribution. Each pixel was described by a five-dimensional vector, and both the intensity and position information of each pixel were considered. Our approach adopted weighted 1-Norm to balance colour similarity and spatial proximity, so that the superpixel compactness was well controlled. The main advantage of the proposed approach was that it provided a quick correct way for generating superpixel segmentation. The performance was evaluated on BSDS500 dataset, and the results revealed that UMMS was a competitive superpixel segmentation approach.

\section{Acknowledgments}

The authors would like to thank the anonymous reviewers and the associate editor for their insightful comments that significantly improved the quality of this paper. This work was supported by the National Nature Science Foundation of China under Grant 61872143, 61771196.

\section{References}

[1] L. Xu, B. Luo, M. Kong, B. Li, and Z. Pei, "Fast superpixel segmentation via boundary sampling and interpolation," IEICE Trans. Inf. \& Syst., vol.E102-D, no.4, pp.871-874, April 2019.

[2] X. Xie, G. Xie, X. Xu, L. Cui, and J. Ren, "Automatic image segmentation with superpixels and image-level labels," IEEE Access, vol.7, pp.10999-11009, 2019.

[3] M.-Y. Liu, O. Tuzel, S. Ramalingam, and R. Chellappa, "Entropy rate superpixel segmentation," IEEE Conf. Computer Vision and Pattern Recognition (CVPR), pp.2097-2104, 2011.

[4] R. Achanta, A. Shaji, K. Smith, A. Lucchi, P. Fua, and S. Süsstrunk, "SLIC superpixels compared to state-of-the-art superpixel methods," IEEE Trans. Pattern Anal. Mach. Intell., vol.34, no.11, pp.2274-2282, 2012.

[5] V. Machairas, M. Faessel, D. Cárdenas-Peña, T. Chabardes, T. Walter, and E. Decencière, "Waterpixels," IEEE Trans. Image Process., vol.24, no.11, pp.3707-3716, 2015.

[6] J. Shen, Y. Du, W. Wang, and X. Li, "Lazy random walks for superpixel segmentation," IEEE Trans. Image Process., vol.23, no.4, pp.1451-1462, 2014.

[7] J. Chen, Z. Li, and B. Huang, "Linear spectral clustering superpixel," IEEE Trans. Image Process., vol.26, no.7, pp.3317-3330, 2017.

[8] Z. Ban, J. Liu, and L. Cao, "Superpixel segmentation using Gaussian mixture model," IEEE Trans. Image Process., vol.27, no.8, pp.4105-4117, 2018.

[9] R.P. Browne, P.D. McNicholas, and M.D. Sparling, "Model-based learning using a mixture of mixtures of Gaussian and uniform distributions," IEEE Trans. Pattern Anal. Mach. Intell., vol.34, no.4, pp.814-817, 2012.

[10] P. Arbeláez, M. Maire, C. Fowlkes, and J. Malik, "Contour detection and hierarchical image segmentation," IEEE Trans. Pattern Anal. Mach. Intell., vol.33, no.5, pp.898-916, 2011. 